\title{
AROMATIC INFRARED EMISSION IN CIRRUS CLOUDS
}

\author{
J. L. PUGeT* \\ Radioastronomie, Ecole Normale Supérieure
}

\begin{abstract}
We discuss the fraction of the infrared cirrus emission radiated out of thermal equilibrium using the IRAS data. Recent spectrophotometric data (Giard et al., 1988a, 1988b) are presented which confirm that aromatic infrared bands account for at least a fraction of their near and mid-infrared cirrus emission as was suspected from indirect arguments. Large variations of the energy distribution among the IRAS bands are shown to be present in molecular clouds. Abundances of polycyclic aromatic hydrocarbons (PAH's) required to account for the emission are discussed.
\end{abstract}

\section{INFRARED CIRRUS}

The name "infrared cirrus" was introduced by Low et al. (1984) for the filamentary structures seen in the $100 \mu \mathrm{m}$ band of IRAS. This emission was shown to be coming from interstellar clouds heated mostly by the diffuse interstellar radiation field (ISRF) (see Puget, 1988 for a review). The analysis of the latitude and longitude distribution and the photometric spectrum of the galactic ridge emission shows that sources (defined as regions where the dust is heated by a strong local stellar radiation field) contribute only $30 \%$ of the galactic emission. Most of the radiation from $O$ and $B$ stars escapes their immediate vicinity and contributes to the ISRF (Leisavitz and Hauser, 1988). HI clouds heated by the ISRF contribute $40 \%$ of the galactic ridge emission and molecular clouds the remaining $30 \%$ (Pérault et al., 1988).

We see that, although of low brightness when observed at high latitude, the cirrus emission is really what makes the largest part of the total galactic infrared emission. The absence of confusion along the line-of-sight when observing cirrus clouds and their rather constant radiation environment make them good places to study the conversion of starlight into infrared emission in our galaxy. We shall discuss cirrus clouds, whatever their molecular fraction.

The average emissivity per hydrogen atom $\left(6.2 \times 10^{-31} \mathrm{~W} / H\right)$ is in good agreement with the expected value (Puget, 1988), but the spectral distribution of this energy among the four IRAS bands does not agree at all with what was predicted by standard dust models. The spectral distribution of the cirrus emission is given

*also at Observatoire de Meudon 


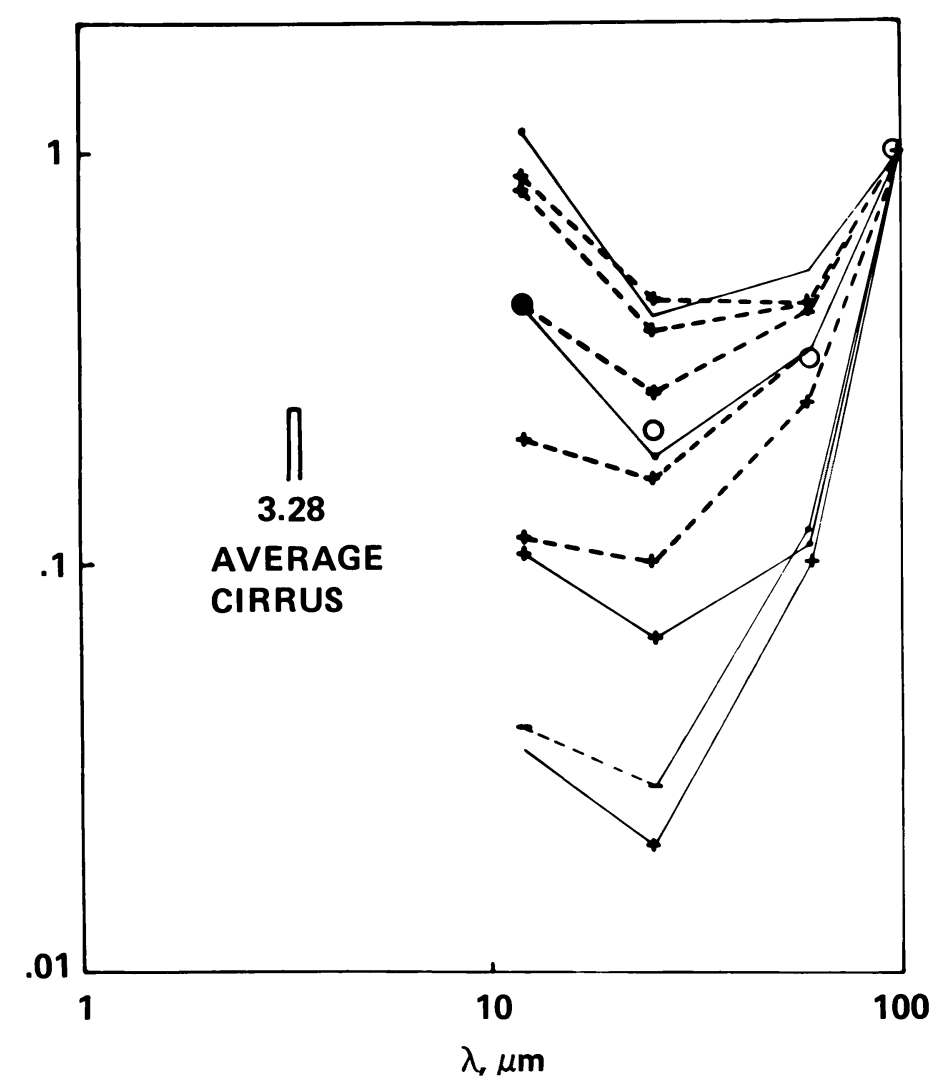

Fig. 1. Distribution of energy among the IRAS bands. The open circles refer to the average cirrus emission. The other distributions refer to various clouds and filaments from the complexes discussed in the text, all normalized at $100 \mu \mathrm{m}$. The $3.28 \mu \mathrm{m}$ feature for the average cirrus emission is derived from the data of Giard et al., $1988 \mathrm{~b}$.

in Figure 1. It was obtained by Boulanger and Pérault (1988) by taking, for the four IRAS bands, the fraction of the high-latitude emission which follows a $\csc (b)$ dependence with galactic latitude $(b)$. The discrepancy between the observed and the predicted intensity in the $60 \mu \mathrm{m}$ band was noticed by Draine and Anderson (1985).

\section{THE AROMATIC INFRARED BANDS IN CIRRUS CLOUDS}

Puget, Léger, and Boulanger (1985) computed the infrared emission for a dust model which included a continuous size distribution extrapolating the Mathis, Rumpl, and Nordsieck (1977) distribution down to a size of about $4 \AA$. The smallest particles in this distribution are polycyclic aromatic hydrocarbons (PAH's) as suggested by Léger and Puget (1984) to be at the origin of the emission of the unidentified infrared bands, and of the excess mid-infrared emission detected along the galactic ridge by 
Price (1981) (see the reviews by Allamandola, 1989; and Léger et al., 1989 for a discussion of this identification). Although the bands have been observed only in the vicinity of strong ultraviolet (UV) radiation sources, the model predicted that cirrus clouds heated only by the ISRF should radiate a rather large fraction of their energy in the mid-infrared through temperature fluctuations of PAH's and should be detectable in the $12 \mu \mathrm{m}$ and $25 \mu \mathrm{m}$ IRAS bands. This mid-infrared emission from cirrus was found by Boulanger et al. (1985). There was, until recently, no spectroscopic confirmation of the chemical nature of the emitters.

The aromatic infrared bands have been extensively studied spectroscopically in regions with high-UV radiation fields (see Bregman, 1989, for a review). Unfortunately the typical brightness of cirrus does not allow spectroscopic observations with warm telescopes. Only ISO or SIRTF will be able to carry out a spectroscopic study of the mid-infrared cirrus emission. A balloon-borne spectrophotometric experiment was built by Giard et al. $(1988 a)$ to measure the contribution of the shortest wavelength aromatic band at $3.3 \mu \mathrm{m}$ in the diffuse galactic emission. The experiment compared the emission in a narrow interference filter centered on the $3.3 \mu \mathrm{m}$ band with that in the continuum measured through a broader filter centered at the same wavelength but excluding the band.

Maps of the galactic emission in the continuum and in the band together with a map of the $12 \mu \mathrm{m}$ IRAS band for comparison can be found in Giard et al. (1988b). A striking feature of these maps is that the continuum, which is likely to be dominated by starlight, is absorbed by large interstellar complexes known to lie in the galactic plane (for example at longitude $30^{\circ}$ ), but the $3.3 \mu \mathrm{m}$ feature emission is enhanced in these directions proving its interstellar origin. In these maps the diffuse emission is clearly seen at a latitude of 3 degrees. The diffuse emission at this latitude drops significantly for longitudes larger than $35^{\circ}$ as the diffuse emission in the IRAS bands does. This has been interpreted as the cirrus type emission of HI clouds lying far above the galactic equator (500 pc) in the inner Galaxy (Puget, 1988; Pérault et al., 1988). To compare the energy radiated in the $3.3 \mu \mathrm{m}$ feature with the emission in the IRAS bands in cirrus and with the total infrared emission, the best is to use this excess at $b= \pm 3^{\circ}$. We deduce from this comparison:

$$
I(3.3 \mu \mathrm{m}) / I_{\text {total }}=6.5 \times 10^{-3}
$$

and

$$
I(3.3 \mu \mathrm{m}) / \nu I_{\nu}(12 \mu \mathrm{m})=2.5 \times 10^{-2}
$$

This ratio is larger than what is observed in reflection nebulae (Sellgren, 1984; Sellgren et al., 1985) or starburst galaxies (Wilner et al., 1977). The ratio of the 12 $\mu \mathrm{m}$ IRAS band emission to the total infrared emission has been found to drop in the vicinity of $O$ and B stars. (Ryter et al., 1987; Boulanger et al., 1988b). This has been interpreted as the signature of the destruction of the PAH's which radiate in the $12 \mu \mathrm{m}$ band. The natural interpretation of the lower ratio of $3.3 \mu \mathrm{m}$ emission to the total infrared emission seen in regions of higher UV intensity than in cirrus, is that the smallest PAH molecules which dominate the $3.3 \mu \mathrm{m}$ emission are destroyed by high-intensity UV radiation and are destroyed more easily than the somewhat larger ones radiating mostly in the $12 \mu \mathrm{m}$ IRAS band. Giard et al. (1988b) mention also that the degree of dehydrogenation of the PAH's might be higher in regions where the UV intensity is higher. 


\section{PAH'S IN MOLECULAR CLOUDS}

The results and discussions found in this section are taken mostly from the work of Boulanger et al. $(1988 a, 1989)$. In the following we call "very small grains" (VSG's) three-dimensional particles small enough to undergo a significant temperature fluctuation when absorbing a single photon. Following the argument of Léger and Puget (1984) we expect most of them to be graphitic just because this chemical form is one of the most refractory. The absence of silicate features in emission in the diffuse interstellar medium or in the integrated spectrum of galaxies excludes a large amount of very small silicate grains (Désert et al., 1986). The graphitic VSG's should be typically made up of 1000 to $5 \times 10^{5}$ carbon atoms, equivalent to spherical grains with radii $15 \AA$ to $100 \AA$. They radiate mostly at wavelengths above $25 \mu \mathrm{m}$. The PAH's are planar molecules made up of, at most, 1000 carbon atoms and radiating mostly below $25 \mu \mathrm{m}$.

To understand the abundances of PAH's and VSG's and the physics of their excitation (and ultimately the origin and chemistry) in the interstellar medium, the molecular clouds are certainly an interesting test case as their various layers are exposed to a rather different radiation field, and relevant chemical and physical processes might take place there (like synthesis of large cyclic carbon molecules in the gas phase or in grain mantles and changes of the size spectrum of interstellar dust grains).

Molecular clouds are not a homogeneous group from the point-of-view of their infrared emission (for both emissivity per unit mass and spectra). The immediate vicinity of sources is out of the scope of this review. We will consider here only clouds heated by the ISRF. The low IR brightness of molecular and HI gas in cirrus clouds restricts - for the time being - such an analysis to the use of the photometric data of IRAS. We will use mostly the $I_{\nu}(12 \mu \mathrm{m}) / I_{\nu}(100 \mu \mathrm{m})$ ratio as a tracer of the energy radiated by PAH's, and $I_{\nu}(60 \mu \mathrm{m}) / I_{\nu}(100 \mu \mathrm{m})$ as a tracer of the energy radiated by VSG's. $I_{\nu}(25 \mu \mathrm{m}) / I_{\nu}(100 \mu \mathrm{m})$ is also given, but the signalto-noise is often rather low, making this color ratio more difficult to use to obtain quantitative results.

Given that the angular resolution of the IRAS survey is rather limited, we restrict ourselves to the nearest clouds situated at galactic latitudes larger than $10^{\circ}$ to minimize the problem of confusion. The IRAS colors of HI clouds are very stable and in agreement with the average colors of the high-latitude emission obtained by Boulanger and Pérault (1988) as can be seen in Figure 2 of Boulanger et al., 1989. The colors of various molecular clouds and filaments in molecular complexes plotted in their Figure 4 show variations of the ratio $I_{\nu}(12 \mu \mathrm{m}) / I_{\nu}(100 \mu \mathrm{m})$ by a factor of more than 30. An extreme case of this color ratio was found by Leene (1986) in a similar environment. For dark clouds these variations are correlated with the variations of the ratio $I_{\nu}(60 \mu \mathrm{m}) / I_{\nu}(100 \mu \mathrm{m})$.

Besides the large dispersion in colors seen between different clouds and filaments discussed by Boulanger et al. (1988), strong limb-brightening or more complicated color gradients are often seen for $I_{\nu}(12 \mu \mathrm{m}) / I_{\nu}(100 \mu \mathrm{m})$ and $I_{\nu}(60 \mu \mathrm{m}) / I_{\nu}(100 \mu \mathrm{m})$ for the PAH's and VSG emissions, respectively, as illustrated by cuts through several clouds and filaments shown in Figure 2 . The data along each scan have been obtained at IPAC using the addscan procedure which adds up individual $2^{\circ}$ long scans. The general direction of the scans displayed is south to north when going from 
negative to positive scan coordinates. Figure 2a shows 3 small clouds in Taurus. The scan is centered at $\alpha=4^{h} 54^{m n} 38^{s}, \delta=+26^{\circ} 42^{\prime}$. One of them at +30 arc min has a very low $I_{\nu}(12 \mu \mathrm{m}) / I_{\nu}(100 \mu \mathrm{m})\left(<1.0 \times 10^{-2}\right)$ ratio when the central one exhibits a value close to the average one for the same color ratio. Using a model of infrared emission from clouds heated by the ISRF, we find that the typical 100 $\mu \mathrm{m}$ brightness of these clouds, 2 to $4 \mathrm{MJy} / \mathrm{sr}$, shows that the total visual extinction through each of them is less than $\sim 1$ magnitude. These clouds belong to the "translucent cloud" type of Boulanger et al., 1989. Another illustration of this kind of color variation in molecular complexes inside small clouds with low or moderate extinction and no UV source in the vicinity is displayed in Color Plate 3. Maps of the emission in band 1 and $4(12$ and $100 \mu \mathrm{m})$ are displayed simultaneously and show striking differences in distribution. (Striping in band 1 is obviously present but does not affect variations along the scan direction). In several cases, the amplitudes of the variations of the color ratio are much larger than can be expected from UV absorption alone for clouds of such opacity.

Figure 2b shows a filament a few parsecs away (in projection) from the $\mathrm{O}$ star $\zeta$ Oph for which images of the emission in the 4 IRAS bands can be found in Boulanger et al., 1989. The scan is centered at $\alpha=16^{h} 44^{m n}, \delta=-9^{\circ} 37^{\prime}$. Very steep gradients of the color ratios are present, with rather extreme values on the southern side. Considering the projected geometry, this side is likely to be receiving UV radiation from $\zeta$ Oph. This example of a filament near the limit of the sphere of influence of a hot star is similar to the case of $\chi$ Per (Boulanger et al. (1988b), where such filaments also show much larger color ratios than their environment.

Figure 2c is a cut through the main filament in $\rho$ Oph for which superposed images of bands 1 and 4 are shown in Color Plate 4. The scan is centered at $\alpha=$ $16^{h} 24^{m n} 16^{s}, \delta=-24^{\circ} 23^{\prime}$. Several effects can be noticed: strong limb-brightening at $12 \mu \mathrm{m}$ similar to what has been seen for the B5 cloud by Beichman et al., 1988 . The minima in the three colors coincide with the peak of the $100 \mu \mathrm{m}$ brightness, which is likely to be close to the peak of column density. An asymmetry is apparent in Color Plate 4, the southern side being stronger at $12 \mu \mathrm{m}$ and being the side receiving radiation from $\tau$ Sco and $\sigma$ Sco (Ryter et al., 1988).

Figure 2d presents a cut through the north east filament in the $\rho$ Oph complex which shows a strong color asymmetry. The scan is centered at $\alpha=16^{h} 39^{m n} 07^{s}, \delta=$ $-24^{\circ} 04^{\prime}$. Once again the three colors are correlated although the relative effect is much smaller at $60 \mu \mathrm{m}$.

Figure 2e presents a cut through the dense core of $\rho$ Oph. The scan is centered at $\alpha=16^{h} 39^{m n} 38^{s}, \delta=-22^{\circ} 01^{\prime}$. One notices that, between the sources, in the densest part of the molecular core as revealed by the $C^{18} O$ data of Wilking and Lada $(1983)$, the $I_{\nu}(12 \mu \mathrm{m}) / I_{\nu}(100 \mu \mathrm{m})$ and $I_{\nu}(25 \mu \mathrm{m}) / I_{\nu}(100 \mu \mathrm{m})$ color ratios fall to very low values (about a factor of 10 below the average), but the more striking feature is the maxima at $\simeq+30$ arc min just outside of the edge of the molecular cloud as detected by its ${ }^{13} \mathrm{CO}$ emission. Further out in the $\mathrm{HI}$ envelope, (from $\simeq$ +30 arc $\min$ to $\simeq+60$ arc $\min$ ) the color goes back down to the average value although the UV radiation field is certainly increasing!

The 3 colors displayed in Figure 2 are often correlated which is a different behaviour of what was found in HII regions by fyter et al. (1987) and Boulanger et al. (1988b). In HII regions, where the $60 \mu \mathrm{m}$ emission is mostly due to big 

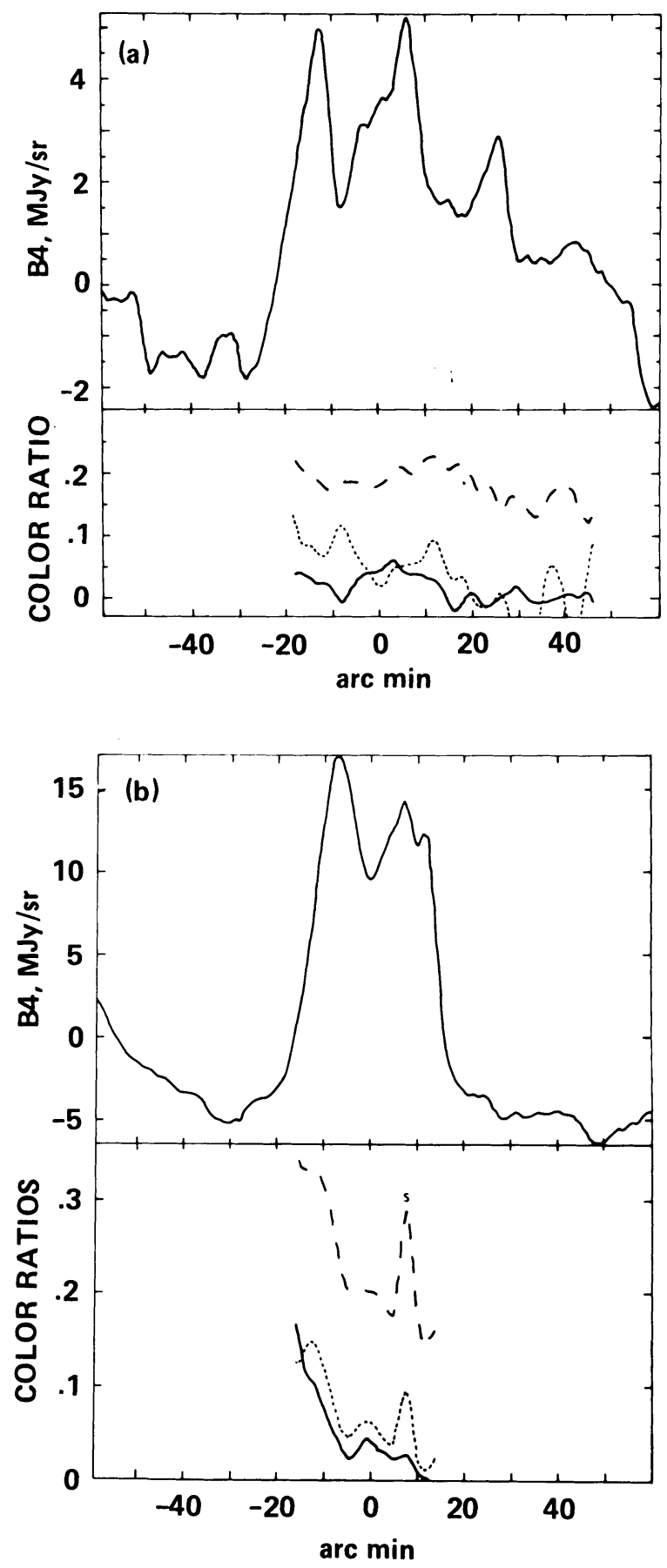

Fig. 2. (a and b) Cuts through a set of clouds. Top: the profiles in band 4 (thick line) is displayed. Bottom: the color ratios $I_{\nu}(12 \mu \mathrm{m}) / I_{\nu}(100 \mu \mathrm{m})$ (full line), $I_{\nu}(25 \mu \mathrm{m}) / I_{\nu}(100 \mu \mathrm{m})$ (dotted line) and $I_{\nu}(60 \mu \mathrm{m}) / I_{\nu}(100 \mu \mathrm{m})$ (dashed line) are displayed in the part of the scan where the signal is strong enough. 

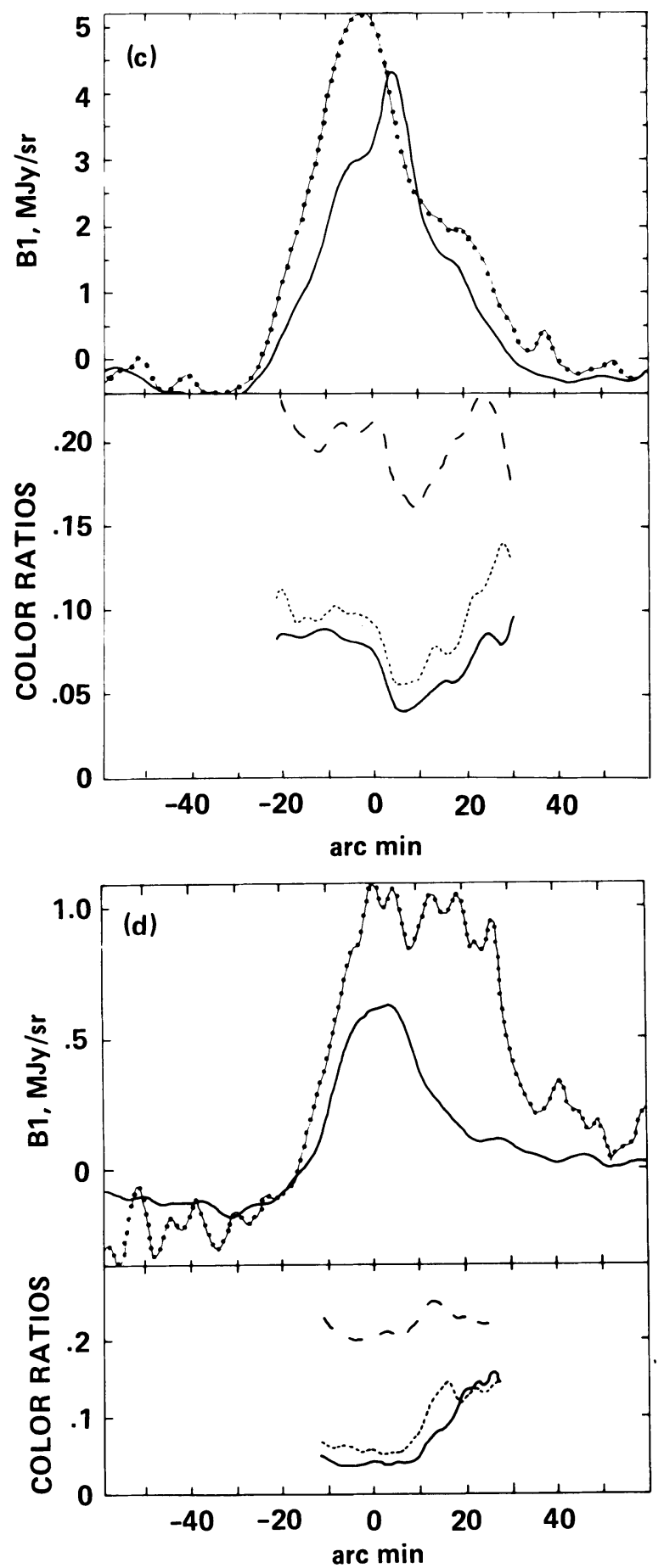

Fig. 2. (c and d) Cuts through a set of clouds. Top: (c) the profiles in band 4 (thick line) is displayed. Bottom: (d) the color ratios $I_{\nu}(12 \mu \mathrm{m}) / I_{\nu}(100 \mu \mathrm{m})$ (full line), $I_{\nu}(25 \mu \mathrm{m}) / I_{\nu}(100 \mu \mathrm{m})$ (dotted line) and $I_{\nu}(60 \mu \mathrm{m}) / I_{\nu}(100 \mu \mathrm{m})$ (dashed line) are displayed in the part of the scan where the signal is strong enough. In addition, the band 1 profile (thin line) is also displayed in the top figure. 


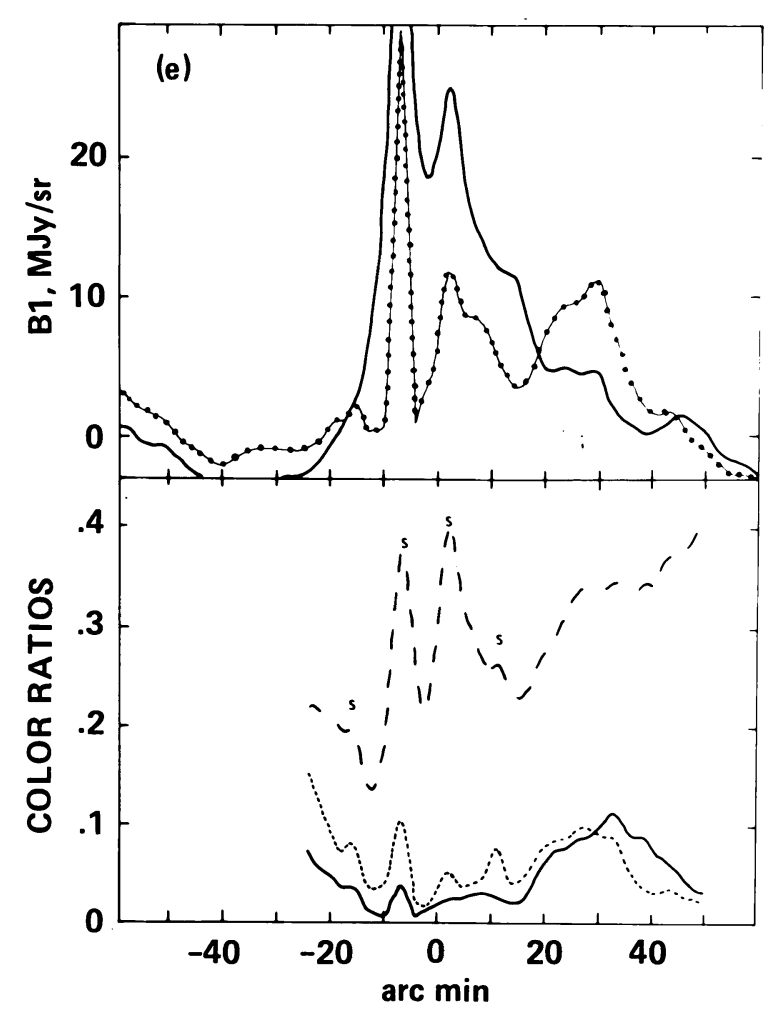

Fig. 2. (e) Cuts through a set of clouds. The profiles in band 4 (thick line) is displayed. The color ratios $I_{\nu}(12 \mu \mathrm{m}) / I_{\nu}(100 \mu \mathrm{m})$ (full line), $I_{\nu}(25 \mu \mathrm{m}) / I_{\nu}(100 \mu \mathrm{m})$ (dotted line) and $I_{\nu}(60 \mu \mathrm{m}) / I_{\nu}(100 \mu \mathrm{m})$ (dashed line) are displayed in the part of the scan where the signal is strong enough. In addition, the band 1 profile (thin line) is also displayed in the top frame.

grains, and the $I_{\nu}(60 \mu \mathrm{m}) / I_{\nu}(100 \mu \mathrm{m})$ is a tracer of the energy density of the UV field, which anticorrelates with the $I_{\nu}(12 \mu \mathrm{m}) / I_{\nu}(100 \mu \mathrm{m})$ color ratio. Laureijs, Chlewicki, and Clark (1988) have pointed out that the $I_{\nu}(60 \mu \mathrm{m}) / I_{\nu}(100 \mu \mathrm{m})$ and $I_{\nu}(12 \mu \mathrm{m}) / I_{\nu}(100 \mu \mathrm{m})$ have different dependence on the opacity of the clouds implying two different small grains populations.

\section{ABUNDANCE OF PAH'S}

Léger (1988) reports measurements of the UV absorption cross-section of several mixtures of PAH molecules of known mean mass. Using the average of these measurements we get, for PAH's molecules immersed in the ISRF a power absorbed of $2.25 \times 10^{-27} \mathrm{~W} /$ Carbon-atom. This value is very close to the evaluation given by Puget (1988). For VSG's we use the cross-section computed by Draine and Lee (1985) for $30 \AA$ graphite particles. The power absorbed by these particles is slightly lower: $1.5 \times 10^{-27} \mathrm{~W} /$ Carbon-atom, the lower value is due to a smaller 
cross-section in the far-UV where these particles start to become opaque. Setting the limit between the wavelength ranges of the emission of PAH's and VSG's (somewhat arbitrarily) at $25 \mu \mathrm{m}$, we can compute the abundances required to account for the average cirrus energy distribution among the IRAS bands. We find that $17 \%$ and $14 \%$ of interstellar carbon should be in form of PAH's and VSG's, respectively.

Beichman et al. 1988 argue that the limb-brightening effect they observed on the B5 cloud can be accounted for only by the variations of the radiation field spectrum as one goes deeper and deeper inside the cloud. This effect is undoubtedly present and accounts for, at least, part of the color variations presented here. Nevertheless, Boulanger et al. $(1988 a, 1989)$ argue that large abundance variations are also required to account for the observations. Arguments to sustain this claim are summarized below. A simple model to discuss the infrared emission of interstellar matter as observed by IRAS can be based on three components (the amount of information from only four photometric bands does not allow a more refined model): big grains similar to the Mathis, Rumpl, and Nordsieck (1977) model but with a reduced amount of graphite particles, PAH's and VSG's with the optical properties described above. This model shows that, for constant abundances of PAH's, the $I_{\nu}(12 \mu \mathrm{m}) / I_{\nu}(100 \mu \mathrm{m})$ color for the line-of-sight going through the edge of a molecular cloud (where the UV field is already significantly attenuated) should be lower by a factor 1.5 from the color in a thin cirrus if the cloud is heated by the ISRF and 1.2 if the cloud is heated by an $O B$ association. The observations of the edges of the $\rho$ Oph molecular cloud core (Figure 2c) which actually show an increase of this color ratio with a maximum just outside the rise of the ${ }^{13} \mathrm{CO}$ emission requires an enhancement of the abundance of PAH's from that of the surrounding HI envelope by at least a factor of 2 .

The large differences in the $I_{\nu}(12 \mu \mathrm{m}) / I_{\nu}(100 \mu \mathrm{m})$ color ratio observed for small isolated filaments in the Taurus complex for which the extinction is rather low cannot be accounted for by absorption of UV alone. The same model shows that a total extinction larger than 10 would be required to explain a $I_{\nu}(12 \mu \mathrm{m}) / I_{\nu}(100 \mu \mathrm{m})$ ratio smaller than $1 \times 10^{-2}$ which is observed in some clouds. But the same model predicts a $100 \mu \mathrm{m}$ brightness larger than about $20 \mathrm{MJy} / \mathrm{sr}$ while that observed is less than $5 \mathrm{MJy} / \mathrm{sr}$, indicating a rather low extinction. The PAH abundance in these clouds must then be reduced with respect to the abundance in the local cirrus by a factor of order 4 .

We conclude that, in molecular complexes, a mechanism modifies the size spectrum of the smallest particles (i. e., changing the relative abundance of PAH's and/or VSG's with respect to the standard interstellar grains). This mechanism should be efficient enough to have a time constant shorter than the mixing time of gas over distances of half a parsec for which large color gradients are observed.

\section{REFERENCES}

Allamandola, L. J. 1989, in IAU Symposium 135, Interstellar Dust, eds. L. J. Allamandola and A. G. G. M. Tielens, (Dordrecht: Kluwer), p. 129.

Allamandola, L. J., Tielens, A. G. G. M., Barker, J. R. 1985, Ap. J., 290 , L25.

Beichman, C. A., Wilson, R. W., Langer, W. D., and Goldsmith, P. F. 1988, Ap. J., in press.

Boulanger, F., Baud, B., and van Albada, G. D. 1985, Astr. Ap., 144, L9. 
Boulanger, F., Pérault, M. 1988, Ap. J., 330, 964.

Boulanger, F., Falgarone, E., Helou, G., and Puget, J. L. 1989, in Interstellar Dust Contributed Papers, eds. A. G. G. M. Tielens and L. J. Allamandola, NASA CP-3036.

- $1988 a$, in preparation.

Boulanger, F., Beichman, C. A., Désert, F. X., Helou, G., Pérault, M., Ryter, C. 1988b, Ap. J., in

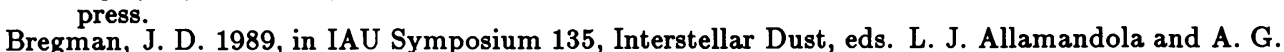
G. M. Tielens, (Dordrecht: Kluwer), p. 109.

Bregman, J. D., Allamandola, L. J., Goebel, J. H., Lester, D. E., Witteborn, F. C., Rank, D. M. 1983, Ap. J., 274, 666.

Cox, P., and Leene, A. 1987, Astr. Ap..

Draine, B. T., and Lee, H. M. 1984, Ap. J., 285, 89.

Draine, B. T., and Anderson, N. 1985, Ap. J., 292, 494.

Giard, M., Pajot, F., Lamarre, J. M., Serra, G., Caux, E., Gispert, R., Léger, A., and Rouan, D. 1988a, Astr. Ap., 201, L1.

Giard, M., Pajot, F., Lamarre, J. M., Serra, G., and Caux, E. 1988b, Astr. Ap., submitted.

Laureijs, R. J., Chlewicki, G., and Clark, F. O. 1988, Astr. Ap., in press.

Léger, A., Verstraete, L., d'Hendecourt, L., Défourneau, D., Dutuit, O., Schmidt, W., and Lauer, J. C. 1989, in IAU'Symposium 135, Interstellar Dust, eds. L. J. Allamandola and A. G. G. M. Tielens, (Dordrecht: Kluwer), p. 173.

Léger, A., and Puget, J. L. 1984, Astr. Ap., 137, L5.

Leene, A. 1986, Astr. Ap., 154, 295.

Leisawitz, D., and Hauser, M. 1988, Ap. J., in press.

Low, F., et al. 1984, Ap. J. (Letters), 278, L19.

Mathis, J., Rumpl, W., and Nordsieck, K. H. 1977, Ap. J., 217, 425.

Pérault, M., Boulanger, F., Falgarone, E., and Puget, J. L. 1988, Ap. J., submitted.

Price, S. D. 1981, A. J., 86, 193.

Puget, J. L., Léger, A., and Boulanger, F. 1985, Astr. Ap., 142, L19.

Puget, J. L. 1988a, in Comets to Cosmology, ed. A. Lawrence, (Berlin: Springer Verlag).

. $1988 b$, in preparation.

Ryter, C., Puget, J. L., and Pérault, M. 1987, Astr. Ap., 186, 312.

Sellgren, K. 1984, Ap. J., $277,623$.

Sellgren, K., Allamandola, L. J., Bregman, J. D., Werner, M. W., and Wooden, D. H. 1985, Ap. J., $299,416$.

Wilking, B. A., and Lada, C. J. 1983, Ap. J., 274, 698.

Willner, S. P., Soifer, B. T., Russell, R. W., Joyce, R. R., and Gillett, F. C. 1977, Ap. J., 217, L121. 